\title{
Astronomy-the Caribbean view from the ground up
}

\author{
Shirin Haque \\ Department of Physics, University of the West Indies, St. Augustine, Trinidad, West Indies. \\ email: shirin@tstt.net.tt
}

\begin{abstract}
The historical development of astronomy in the Caribbean is reviewed within its cultural and environmental framework. The present status of astronomy in education, research and at the popular level is presented also with the focus being on its development in the island of Trinidad and Tobago in particular. We review what works in small developing islands versus larger developed or developing countries and the peculiar trials and tribulations of our circumstances as well as the rewards of such efforts. The critical role of students and volunteer effort will be highlighted. The psychological and cultural aspect and its role in the development of astronomy in the Caribbean is also explored. The outlook for the next decade will be highlighted with a brief proposal of having a node for TWAN (Third World Astronomy Network) in Trinidad in the Caribbean.
\end{abstract}

Keywords. Astronomy in the Caribbean, Trinidad and Tobago

\section{Introduction}

The Caribbean islands and in particular Trinidad and Tobago have been known on the world scene for their carnival, music, and Miss Universes. The islands have produced Nobel laureates and made it to the World Cup in football but little is known if anything regarding astronomy in the Caribbean. This paper hopes to alleviate the misconception that there is no astronomy activity in the Caribbean. Astronomy exists in the Caribbean in all facets of education, research and popular outreach.

The history of astronomy is closely linked with the history of the University of the West Indies and the islands. The history of the Caribbean is one of slavery and indentured labour. With such a history, there was suppression of creativity and independent thought for hundreds of years in this region. Independence came to the Caribbean islands around the 1960s and with it the emergence of the University of the West Indies.

\section{Astronomy at the University of the West Indies}

The University of the West Indies (UWI) is one of the few regional universities in the world. Its three campuses are located respectively on the islands of Jamaica (Mona), Barbados (Cavehill) and Trinidad (St Augustine). Physics department was established at first at the Mona campus in Jamaica with areas of research in photometry and radio astronomy. Professional astronomy existed and continues to exist at St Augustine and at Mona campuses. Astronomy was established at Mona in the 1970s and at St Augustine from 1978 onwards. At any given time since, there have been 1-2 astronomers at the Mona campus and one at the St Augustine. At Cavehill in Barbados, occasionally there is an astronomer but there is no regular faculty position.

Mona and St Augustine have had taught programmes and research activity. At both campuses, the taught programme is relegated to one course in the undergraduate programme counting towards the physics major at the University. At both campuses, there 
has been an average of 1-2 postgraduate students pursuing astronomy over a ten-year period.

Observational astronomy was present at the Mona campus where they used a 21-inch telescope located at Stony Hill. However, this was damaged during some hurricanes and subsequently, due to increased crime problems and a downturn in the economy, it was never repaired or replaced. Astronomy at the campus there has been on a downslide due to the above-mentioned problems and deaths and migration of faculty reflecting also a general downturn in the physics department as a whole.

At the St Augustine campus, there was only research in theoretical astronomy at the start in areas of cosmology and quasars in the early 1980s. Up to the present, there are several research projects that undergraduate students perform each year as part of their degree programme. Lately, the size of the astronomy class has grown to almost 70 students from about 30 students in the past, in the advanced part of the programme.

However, around 2000, the St Augustine campus launched on an observational programme in conjunction with Tuorla Observatory, University of Turku in Finland. UWI now has access to 40-cm Meade telescope located at the University campus at St Augustine. This facility is known as the SATU (St AugustineTuorla) observatory. The primary project it is dedicated to is the monitoring of the variability of quasar OJ287. Our efforts have yielded a couple of publications in the Astrophysical Journal already.

Presently astronomy at the St Augustine campus is thriving and is on a growth path with three postgraduate students currently pursuing research in the areas of theoretical and observational astronomy, and astrobiology. In small countries, it is important to exploit all natural resources and Trinidad is home to one of the few natural pitch lake sites in the world. This is literally a hydrocarbon pool and we are examining it as an analogous site to the hydrocarbon lakes on Titan and the possibilities of the existence of extremophiles in such a condition. The astrobiology project is in conjunction with collaborators from Washington State University, Villanova University and University of British Columbia.

\section{Education}

Astronomy forms a small part of the primary school curriculum and is no longer in the high school curriculum since the Caribbean moved away from the Cambridge A-level exams and has its own equivalent examination. However, several schools have astronomy clubs that our groups assist with. As indicated in the previous section, it is part of the degree programme in Physics at the University of the West Indies and there are students doing postgraduate work in astronomy.

\section{Amateur astronomy}

It is interesting to note that the astronomy societies in the Caribbean exist on the three campuses where the University campus is located and in every case, the Astronomy Society predates the professional astronomy in the island. The Trinidad and Tobago Astronomical Society was formed in 1965 and the Barbadian Astronomical Society celebrated its 50 th anniversary in 2006 . The societies meet monthly and have viewing sessions and lectures. They own some 12-inch and smaller telescopes. We have conducted several nationwide surveys to gauge the interest in astronomy in schools. The results consistently show a high level of astronomy interest among high school students, but the teachers lack confidence in teaching in this subject area. 
In 2002, there was the emergence of CARINA (Caribbean Institute of Astronomy) based in Trinidad, whose mandate is to unite and promote the growth of astronomy in the Caribbean as a whole. CARINA has been involved in running workshops and outreach programs in Trinidad and in the other islands. In addition, on the island of Tobago, there is a privately owned observatory SEAS (Sea, Earth And Sky) by an eye specialist and astronomy enthusiast, Dr Bruno Mitchell. CARINA is involved in running and overseeing its operation and the University has access to it as well. It currently houses a 12-inch telescope but plans are afoot for a 20-inch telescope to be put there.

\section{Outreach}

One of the popular annual events in Trinidad has been the star party run by CARINA. This event is sold out every year to capacity with over 400 persons attending the event this year. It is interesting to contrast the attendance at star parties versus attendance at astronomy seminars in the Department of Physics, which are held in comfortable environments, at convenient times with refreshments, versus star parties at distant locations and demanding hours where an entrance fee is charged and no seating is provided. The author suggests that the success of star parties versus seminars directly reflects basic innate human characteristics and a certain need-satisfaction mechanism. As part of the outreach programme, seminars and open public lectures and viewing sessions in astronomy are regularly held. This is often in conjunction with the National Science Center located in Trinidad that also has an astronomy popularization programme.

\section{Cultural astronomy}

Cultural astronomy deals with the disseminating and educating the population on aspects of astronomy of religious relevance to them in determining their festivals. The three main religious denominations in the Caribbean are Christianity, Islam and Hinduism. All rely on some aspect of astronomy in determining their festivals. Towards this end, a massive effort has been made in particular with the Muslim community that uses a lunar calendar and the sighting of the new moon to determine their festivals. This drive has been very successful in educating the nation to astronomical aspects of the lunar calendar.

\section{Challenges in the Caribbean}

Some of the problems on the ground are cultural, for example, email culture is not fully developed, although the technology is fully here. There is a serious lack of human resources and funding is often an issue as astronomy is not necessarily seen as a priority in the needs of the Caribbean people by decision makers. Those that are active in the field invariably become jack-of-all-trades as there is lack of infrastructure and to get any project underway one must play a multidisciplinary role. For such reasons, things generally take much longer to get done in the Caribbean than would take place in a developed nation. What could take a few months could easily take a year or two to pull together in the Caribbean due to lack of infrastructure and human resources. But the challenges can be overcome to an extent as we see in the discussion section. 


\section{Discussion}

The small island plight is different from that of a larger developing nation. For us it is a numbers game. The population of Trinidad and Tobago is around 1.2 million, and Jamaica is around 2.7 million, with the other islands being in the order of $100000 \mathrm{~s}$. With such numbers, the absolute number of professional astronomers will always be small and therefore systems in place will be small, which becomes a vicious cycle arresting the development of astronomy in such regions. However, today it is possible to overcome such limitations to an extent, thanks to the internet age, where our colleagues may not be there in real space but are just one click away for consultation. Collaboration is critical for small islands with institutions in other countries. Volunteer student effort is also critical in such situations for the successful progress and growth of astronomy. It is humanly impossible for one astronomer to do it all. It is thus important for students to gain exposure to the international community and towards this end, our undergraduate students have participated in astronomy research in Germany and La Palma, Canary Islands. Three of our students took part in the ISYA summer school in 2005 when it was held in Mexico.

Our thesis for the growth of astronomy in the Caribbean is one of mixing business with pleasure. And it comes from the answer to the question of why seminars are so poorly attended while star parties are heavily supported. The latter evokes the adventurous spirit and having fun and intellectual excitement - innate needs in persons. Seminars may provide intellectual stimulation but there is no fun and adventure in it in general. Tourism forms a big part of the economy of the Caribbean for its natural sun and sand, ecotourism and delightful cultures. This we think can be a motivator to attract astronomers to this part of the world. The greatest number of academic visitors to our department of Physics has been in the area of astronomy in the last few years. And visiting Astronomers initiate collaboration and give public lectures.

There is much discussion about the idea of the development of TWAN (Third world Astronomy Network). We would like to suggest, that although it may sound farfetched since the astronomy group seems so small in the Caribbean that, the Caribbean region and in particular Trinidad, be considered as a possible node for TWAN. The presence of the University campus provides the infrastructure and support that such a node would require and it would attract visitors which would act as a major impetus to the growth of astronomy in the Caribbean. The favourable aspects are that Trinidad is at latitude of about 10 degrees north, assuring a very large amount of skies. It is a country having an oil based economy that is doing well and Trinidad is listed as one of the safest places in the Caribbean during the hurricane season. In addition, English is the language spoken and there is total connectivity here. Also, the location of Trinidad is quite central to about three- quarters of the world - North America, South and Central America, Europe and Africa.

We hope in the next ten years or so, that astronomy grows and has a wider presence across the Caribbean in the areas of outreach and research in the non campus islands as well. The growth of online education can further facilitate this process. We hope to implement a course on astronomy for non science majors at the University of the West Indies. A feasibility study is currently underway to establish a radio telescope in Trinidad. We hope that the possibility of the Caribbean as a TWAN node may be considered. 


\section{Conclusion}

Small developing islands are different from large developing countries. We have difficulties and opportunities that are different to the other developing nations and a lot of it is very much the numbers game - smaller populations by its very nature have reduced number of professionals and expertise. Some of the lessons learnt from our experience are that small developing islands must do small manageable projects. In astronomy, everything needs doing - It is not as important as what you are doing - but that what you do, you should do it well, do it right and achieve results. It takes a lot of dedicated effort but with the assistance of colleagues in developed nations, volunteer student effort, no task is impossible once the endeavour is passion driven. At the present time, in the Caribbean, often, the direct fruits of our labour are intangible and simply satiate the higher calling of the love of the discipline of astronomy, and that is reward enough.

\section{Acknowledgements}

The author would like to thank Dr Dipak Basu, former astronomy faculty at St Augustine, UWI and Dr Maura Imbert, President of the Trinidad and Tobago Astronomical Society for information

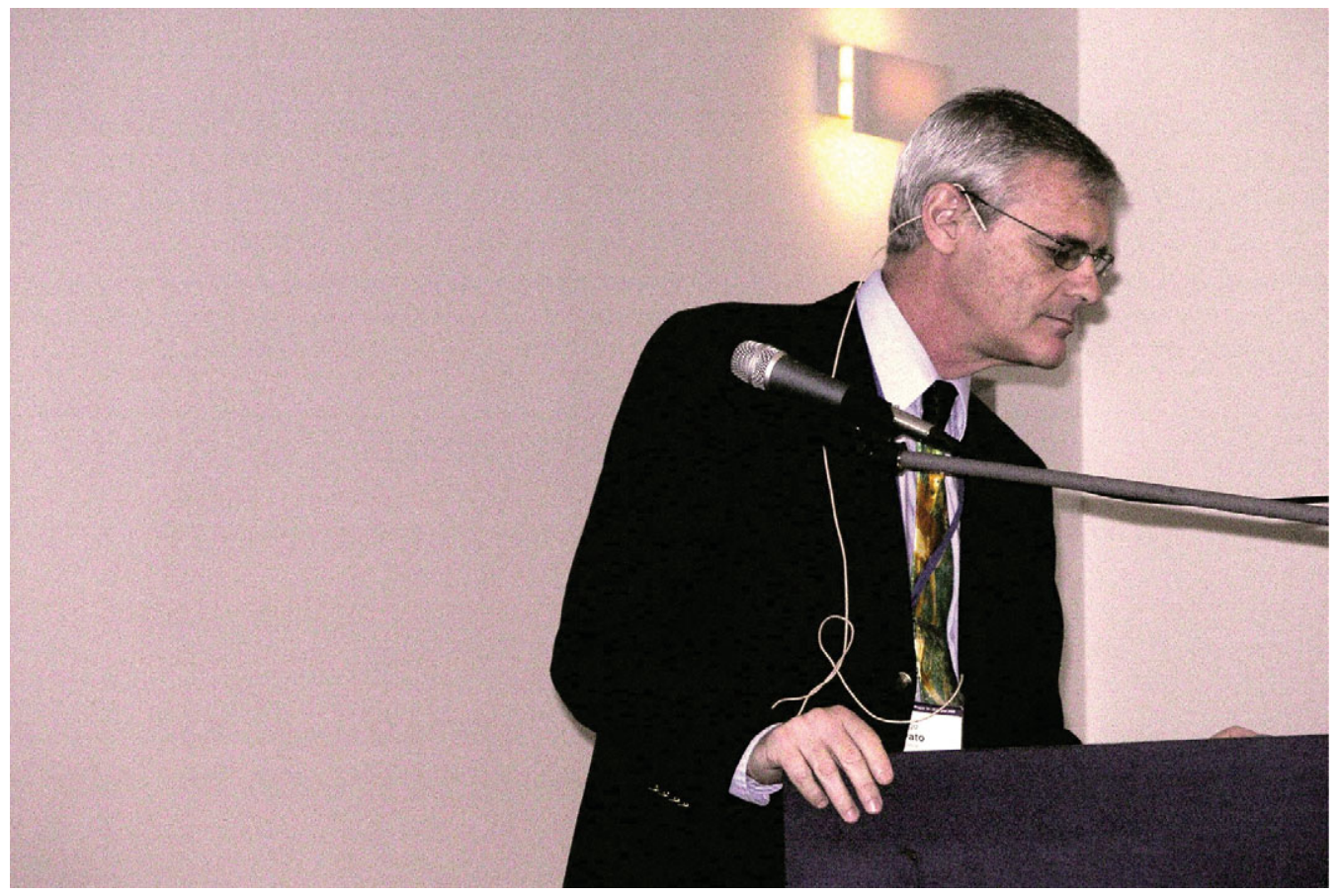

Hugo Levato 


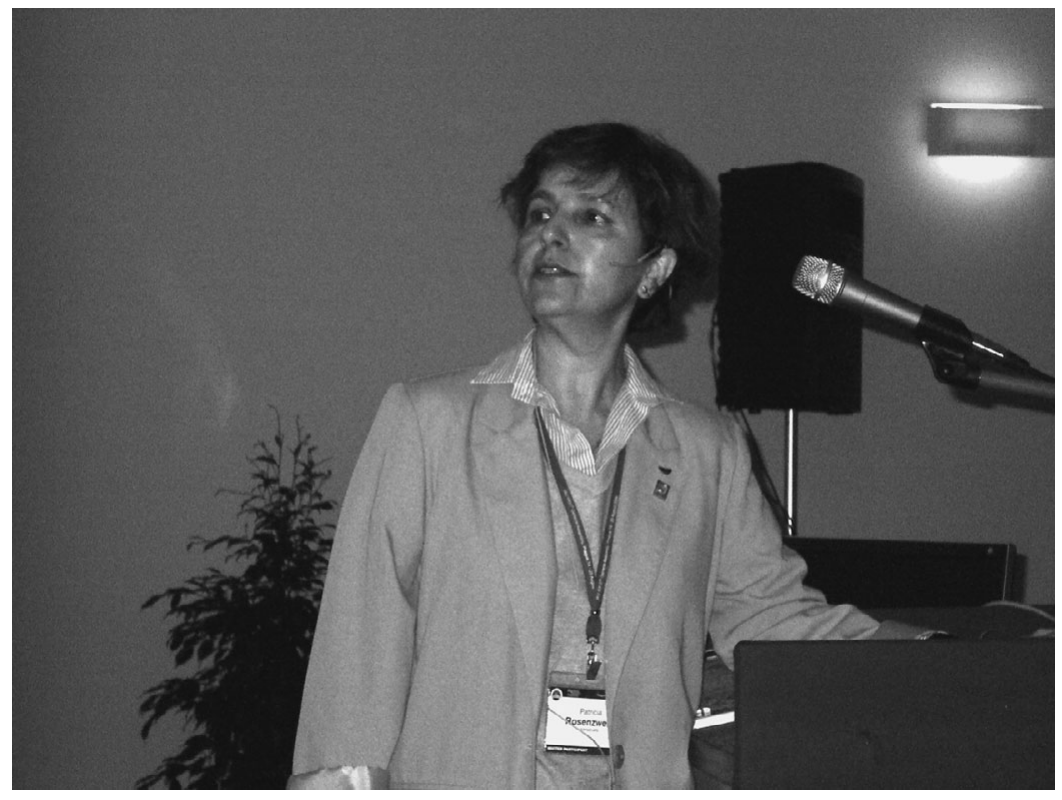

Patricia Rosenzweig

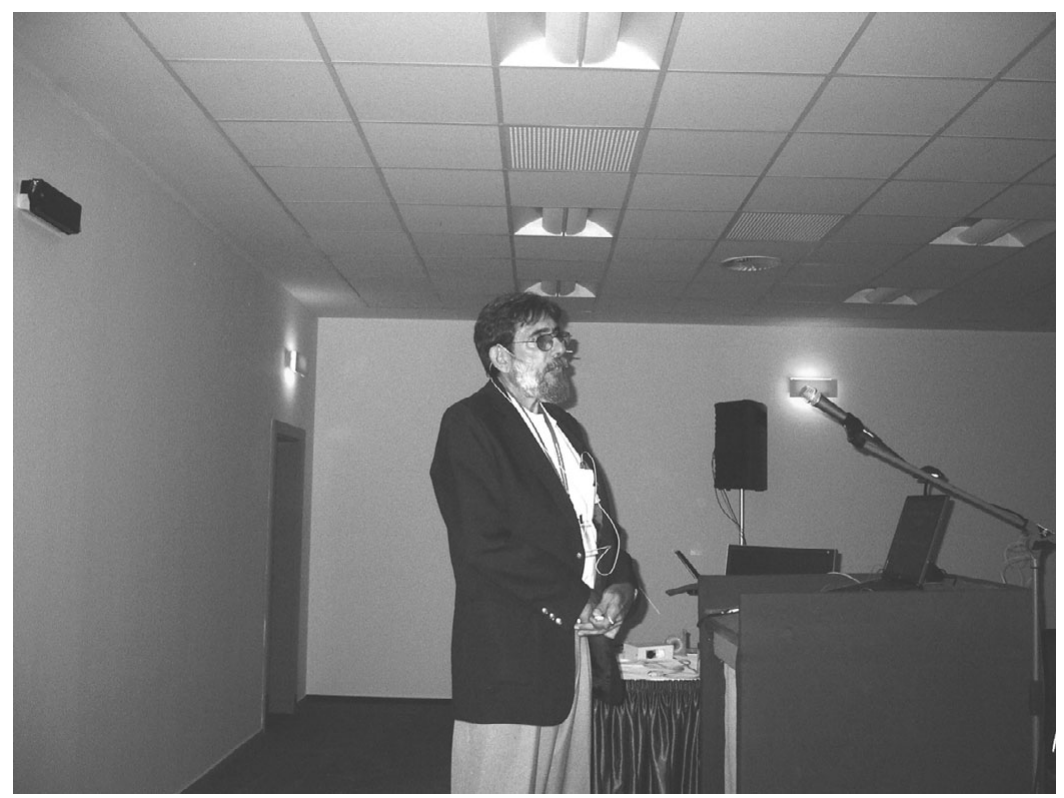

Ramón Rodríguez 